\title{
Intelligents Pillbox: Automatic \& Programmable Assitive Technology Device
}

\author{
Hrushikesh Parkar ${ }^{1}$, Sagar Bhatiya ${ }^{2}$, Abhishek Nadhe ${ }^{3}$, Dr. Prakash M. Mainkar ${ }^{4}$ \\ Electronics \& Telecommunications Department, \\ Maharashtra Institute of Technology, Pune
}

\begin{abstract}
There can be a lot of individuals who need constant help may it be our elderly people, family members, the ones who have special needs. These people apparently need the care which most family members cannot provide due to their busy schedule. Some people may forget to take the medicines at the correct time and can forget the medicines which they have to take. So, in order to help them with this liability we have developed this project for hospital application. In hospital the people are provided a Pill box on which there will be a display which notifies the people about the medicine. Along with this we can alert them with an alarm. So that even if the patient is sleeping or busy with some work the alarm helps in alerting him. To confirm that the patient has taken that medicine or not we can put IR Sensor at the opening end of the pillbox. So, when the alarm gets on the automatically the pill box gets open using pill dispenser mechanism. When the patient put his/her hand in the box the IR Sensor Recognized and the alarm will be off automatically. By this data we can tell that the patient has taken the medicine. It comes with one more feature that when the patient is taken the medicine intimate to the related doctor by using Think speak server. By this system helps patients to take the required medicine in the right quantity at the right time without need of any nurse in the hospitals. If the patient is physically disabled and not able to move and take the medicine for such patients, we are using android application so that they can control the robot movement and can take their medicine in time without taking the help of any other person. Also our pill box contains BP and heart rate monitoring sensor to notify hospital management for quick treatment at critical condition.
\end{abstract}

\section{INTRODUCTION}

As the cost of home medical service rises, it has become more and more incumbent upon individuals in need of supervised medical care to find a means to lower their medical care costs. As such, many individuals who require the dosages of medications at specific times have turned to devices such as automatic pill dispensers to alleviate the need for an in-home nurse on a daily basis. These dispensers' range in cost from $\$ 200$ up to $\$ 800$. These dispensers allow for an in-home medical care provider to have a means to regulate a patient's medications without having to constantly supervise the patient. Typical features of these dispensers include automatic pill dispensing at regular intervals, audible warnings, as well as a connection to a phone for monitoring purposes by the medical care provider. As previously mentioned, many of these devices are expensive and can be somewhat cumbersome. By using a simple microprocessor and motor unit an automatic pill dispenser can be produced for a much cheaper price and be much more user friendly.

The team will design and build a pill dispenser. The product will consist of a motor with 22 fan-like blades that rotate about the central axis. They will form the compartments where pills can be manually placed for dispensing at predetermined times.
The dispenser will be controlled by a microprocessor that is connected with an LED display, as well as an alphanumeric keypad that will be utilized as a source for the inputting of data, and selecting from pre-programmed menu items. The user will be able to input the time(s) of day that pills will be dispensed, as well as any warnings and/or precautions that must be followed when the patient takes his or her pills. Lastly, the dispenser will visually and audibly notify the patient about the pills dispention, and will also store the time of day when the patient took his or her medications. Lateron, the dispenser will automatically adjust the time of the next medication dispersal if necessary, to avoid dosages of medication being taken too closely together. The project will be realized with the development of a compact, pill-dispensing unit that can be placed on a table or counter. A microprocessor unit will control the form and function of the device according to specifications

\section{BLOCK DIAGRAM \& DESCRIPTION}

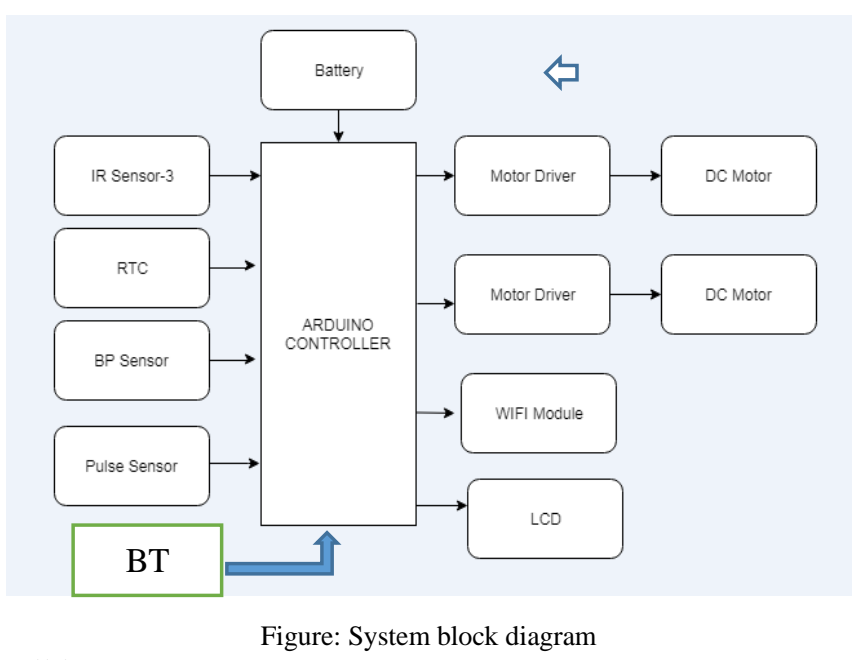

Pill box section

In this Pill box section consists of Arduino controller, IR Sensors, battery, Wi-Fi module, LCD, DC motor and motor driver. Here IR Sensor is used for identifying the box open or not. It is interfaced to the controller by using pins. RTC module is used for identifying the time and date it is interfaced to the controller. Based on this Time and Date automatically updated. Pill box will be opened automatically by using DC Motor via motor driver interfaced to the controller. All the information regarding medicine taken or not status updated to the monitoring person by using Think speak server. BP sensor used to monitor blood pressure pulse sensor to monitor cardiac readings for heart. As the BP ECG goes to critical level system will notify to hospital management 


\section{Mobile Section}

Mobile application is used for controlling the robot movement. Firstly the proposed system gets the hour information from the Real time clock (RTC) and compares with saved hour in controller in order to create a specific alarm for each dose of medicine .This device includes a box with different compartments in order to help the patient to take the correct dose of medicine from the pillbox. It is activated when the door opens, in addition to this; LCD display is used for displaying the medicine taken or not status. As per the saved time in controller when the alarm gets on time then with the help of motor the box which contains pills will get open automatically. The alarm activates the notification system that sends an SMS through the think speak server to the concerned doctor in order to remind them of taking the medicine. If the patient takes the medicine, we get message "MEDICINE TAKEN ON TIME ".and if the IR sensor doesn't receive any signal after 3rd time alarm activation then an alert message "MEDICINE not taken" is sent to the concerned doctor through Thinks peak server. In our project the alarm gets three times with a few seconds gap. On first alarm if the patient takes the medicine then other alarms will not get on but if the patient did not take the medicine at first alarm then pill remainder will be given to the patients 2 more times.

\section{Pulse Sensor}

This is a Heart Attack Detection sensor by Heart Rate Monitoring system helps to inform if a person is about to have a heart attack. This system does this by sensing the heart beat level and informs as soon as the heart beat level drops below the permissible limit. Thus, this system will alert the doctor about the patient's heart beat level. The system uses two circuits i.e. one circuit which is attached to the patient and the other which is being supervised by the doctor or nurse. The system makes use of pulse sensor to find out the heart beat level and display it on the LCD screen.

\section{Blood pressure}

The Blood Pressure Sensor is a sensor designed to measure human blood pressure. It measures systolic, diastolic and mean arterial pressure utilizing the oscillometer technique. Pulse rate is also reported. Blood pressure (BP) is nothing but the pressure of circulating blood on the walls of blood vessels. Most of this pressure is due to the work done by the heart by pumping blood through the circulatory system. Used without further specification, \& quot; blood pressure \& quot; usually refers to the pressure in large arteries of the systemic circulation.

\section{Bot movement section}

Bot section consist of Arduino IC, Bluetooth module, DC driver module, $2 \mathrm{dc}$ motors. If the bot is away from patient then the patient can move the bot using android mobile. Movement of bot is controlled using mobile application via Bluetooth. When patient want a bot near him/her then he/she just have to use mobile application provided to bring bot near him/her. The movement is carried out using mobile Bluetooth and receiver Bluetooth module which then send signal to Arduino IC which then send signal to de driver module and then according to that dc motors performs a respective movement.

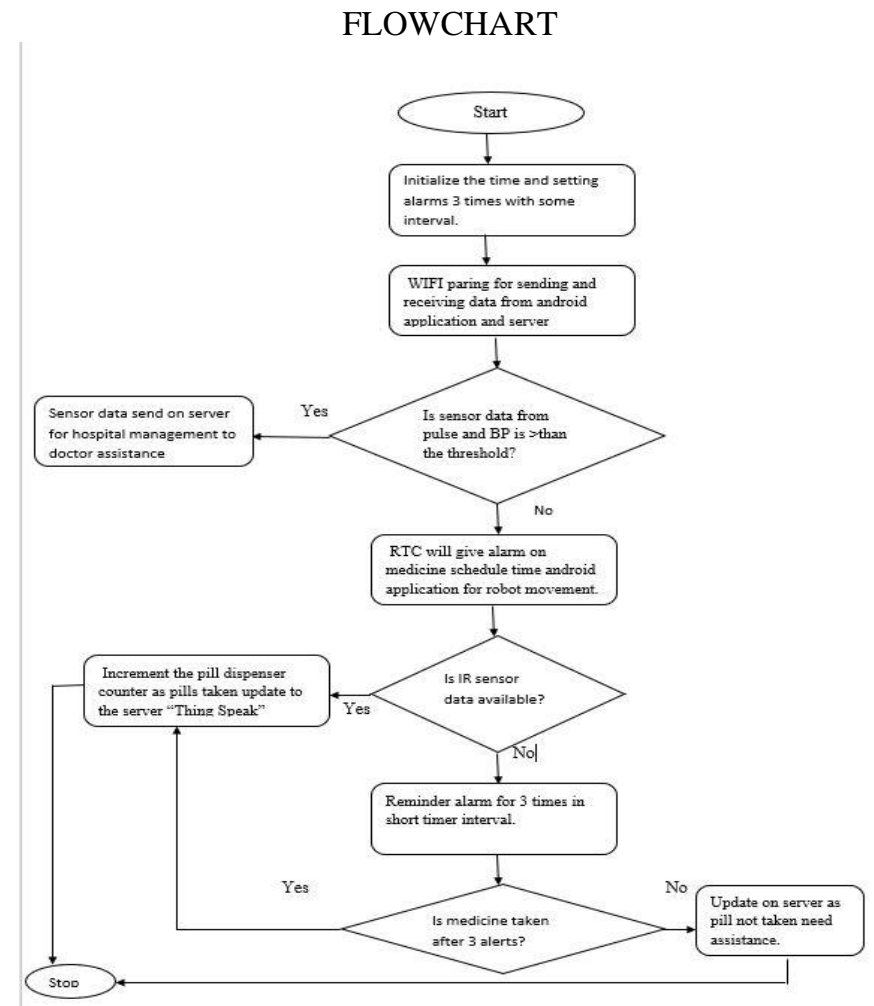

IMPLEMENTATION OF SYSTEM

\section{Algorithm}

1) The Robotic BOT will go near to patient through Android App.

2) Then the Patient will take the Pill from Pill Dispenser.

3) The IR Sensor will detect the presence of patient that will take the Pill or Not.

4) If Patient is not taking Pill, the BOT will give two more chance to give the pill.

5) But after this if patient is not able to take pill, then BOT will send notification to Admin.

\subsection{Algorithms of Code-}

1) Install the Libraries of RTC, Display, Software Serial, etc.

2) Read the values of IR sensor.

3) According to the value of IR sensor Arduino will decide either Patient take pill or not.

4) According to that the data will be sending towards cloud.

5) And Admin take appropriate decision.

\section{EXPERIMENTAL IMPLEMENTATION AND RESULTS}

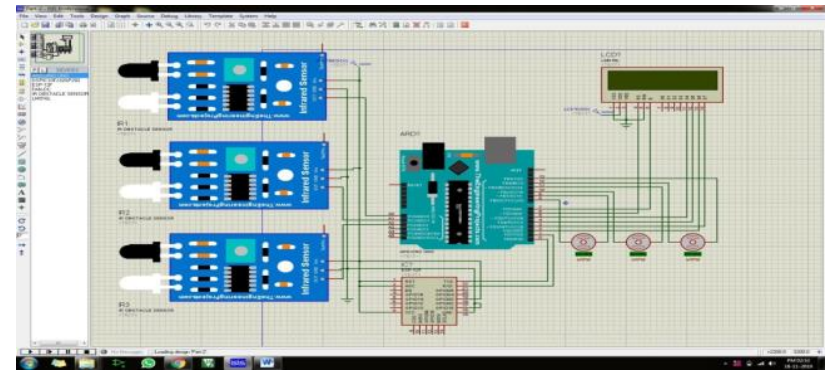

Fig: Simulation of pill section 


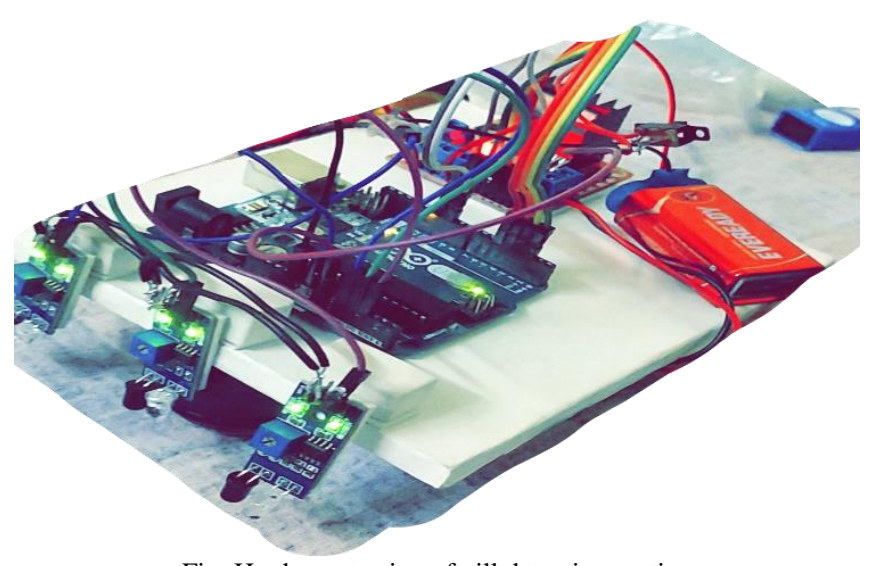

Fig: Hardware testing of pill detection section

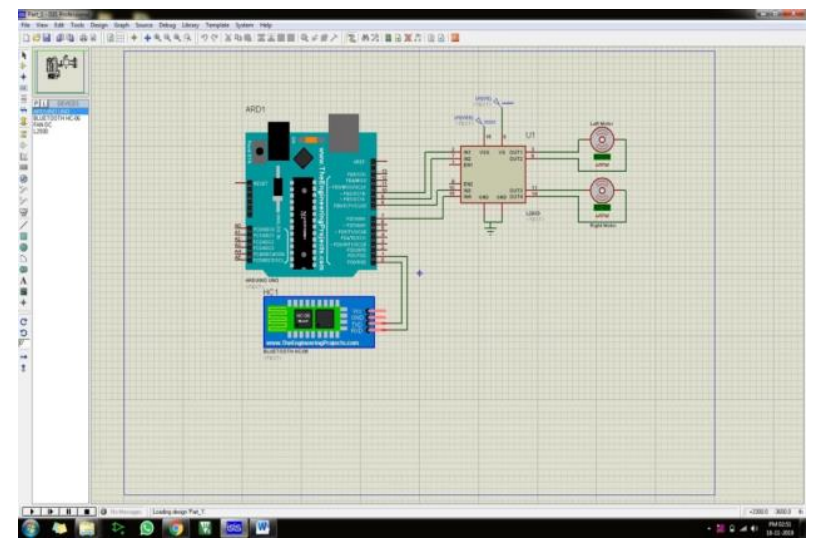

Fig: Simulation of BOT section

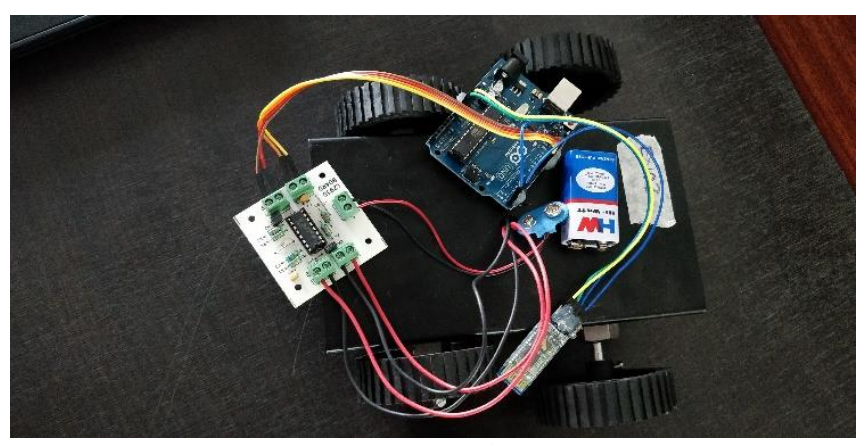

Fig: Hardwar testing of BOT section

\section{CONCLUSION}

The proposed system is made to help the old age patient especially who used to forget to take medicines on time or couldn't recognize / read the name of the medicines. It has the facility to send alarms four times a day. In case patient doesn't take medicines even after alarm then GSM system will send a message to the particular number fed in the system memory. It is possible to program in order to change the number of times dispensing the medicines as per requirement.

\section{FUTURE SCOPE}

In the future, we hope that the application can be to linked to pharmacy so that if the compartments having tablets are empty it directly sends a prescription message to the pharmacy in which they can help us delivering the prescribed tablets to our door step. Scanning of prescription to load the app can also be done using image processing technology.

\section{ACKNOWLEDGEMENT}

We would like to thank our research paper guide Dr. Prakash M. Mainkar for his valuable guidance and support for making this project a success. We submit this paper with great humanity utmost regard.

\section{REFERENCES}

[1] A Smart Pill Box with Remind and Consumption Confirmation Functions Authors-Huai-Kuei Wu1, Member, IEEE, Chi-Ming Wong2, Pang-Hsing Liu1, Sheng-Po Peng1, Xun-Cong Wang1, ChihHi Lin1 and Kuan-Hui Tu1

[2] The Intelligent Pill Box - Design and Implementation AuthorsShih-Chang Huang, Hong-Yi Chang*, Yu-Chen Jhu, Guan-You Chen

[3] Smart Medicine Dispenser (SMD) Authors-Wissam Antoun, Ali Abdo and Suleiman Al-Yaman Department of Computer and Communication Engineering, Faculty of Engineering, Notre Dame University-Louaize

[4] The Autonomous Pill Dispenser with Alarm and Mobile Notifications Authors-Jayesh Patil, Sameer Khairmode, Rishikesh Lokhande, Omkar Shinde Computer Department, PVG's College of Engineering and Technology Pune.

[5] Pill Dispenser with Alarm Via Smart Phone Notification AuthorsNurmiza Binti Othman and Ong Pek Ek Biomedical Modelling and Simulation Research Group, 1,2Faculty of Electrical and Electronic Engineering, University Tun Hussein Onn Malaysia, Parit Raja, 86400 Batu Pahat, Johor, Malaysia

[6] https://www.pivotell.co.uk/downloads/WMPDReport.pdf

[7] https://www.epill.com

[8] https://www.researchgate.net/publication/321110414_Automatic_Pil 1_Dispenser

[9] https://courses.engr.illinois.edu/ece445/getfile.asp?id=11908

[10] https://courses.engr.illinois.edu/ece445/getfile.asp?id=11908 